\title{
Application of Polynomial Mathematical Models for the Extraction of Bioactive Compounds from Plant Sources
}

\author{
Mariana Rusu', Aliona Ghendov-Mosanu2*, Rodica Sturza ${ }^{3}$ \\ ${ }^{1}$ Department of Applied Informatics, Technical University of Moldova, Chisinau, Republic of Moldova \\ ${ }^{2}$ Department of Food Technology, Technical University of Moldova, Chisinau, Republic of Moldova \\ ${ }^{3}$ Department of Oenology and Chemistry, Technical University of Moldova, Chisinau, Republic of Moldova \\ Email: *aliona.mosanu@tpa.utm.md
}

How to cite this paper: Rusu, M., Ghendov-Mosanu, A. and Sturza, R. (2021) Application of Polynomial Mathematical Models for the Extraction of Bioactive Compounds from Plant Sources. Applied Mathematics, 12, 1126-1144.

https://doi.org/10.4236/am.2021.1211072

Received: October 18, 2021

Accepted: November 27, 2021

Published: November 30, 2021

Copyright (c) 2021 by author(s) and Scientific Research Publishing Inc. This work is licensed under the Creative Commons Attribution International License (CC BY 4.0).

http://creativecommons.org/licenses/by/4.0/

\begin{abstract}
This article focuses on the mathematical modelling of the extraction process of bioactive compounds from grape marc and berries (Aronia, rosehip, rowan, and hawthorn). The composition of the extraction medium (the concentration of the ethyl alcohol) served as a factor of influence. Furthermore, 8 experimental measured parameters were used as variables. The experimental results were processed using Hermite polynomials. In order to adapt the degree of the polynomial, the following conditions were imposed: high precision of the mathematical model by appealing to models on interval; obtaining a nominal model and two uncertain models (upper and lower); deduction of two predictive models, one superior and one inferior. It was found that the mathematical models based on Hermite polynomials do not provide explicit analytical expressions, although they allow the establishment of parameter values for any concentration of the extraction medium. In some cases, only high-grade polynomial models ensure the modelling error below $2 \%$. Uncertain models (upper and lower 95\%) include all experimental data. Predictive mathematical models (upper and lower) were established for a high prediction. The analytical expressions of the mathematical models on intervals are non-gaps, the coefficients having non-zero values. Dependencies between the measured parameters and the composition of the extraction solvent were analyzed, the results being presented through the calculation of a surface, with all the experimental values and their average values. Thus, it was found that polynomial mathematical models provide complete information for modelling the extraction processes of bioactive compounds of plant origin.
\end{abstract}

\section{Keywords}

Polynomial Mathematical Models, Error, Upper and Lower Uncertainty 
Model, Ethyl Alcohol Concentration, Parameter, Plant Sources

\section{Introduction}

The number of the experiments that can be held during research is limited, because of certain causes, including economical aspect. Thus, in all fields, for the theoretical study of a certain process, the mathematical model is established, an algorithm that describes the evolution in time or after certain mutual dependencies between quantities. When establishing the mathematical model, simplifying hypotheses are adopted and approximations are made on the parameters of the analyzed process. The hypotheses adopted and the approximations made lead to incomplete mathematical descriptions that are far from reality. For these reasons, the establishment of the mathematical model (possibly) is frequently prefigured theoretically and then finalized on the basis of experimental data [1]-[6]. The analysis of the experimental data highlighted the existence of more or less accentuated nonlinear dependencies; for this reason, mathematical models should be nonlinear, the most widely used are polynomial models. Polynomial models may or may not provide explicit analytical expressions. The aim of this paper is to apply polynomial mathematical models that provide explicit analytical expressions to obtain full information for modeling the extraction processes of bioactive compounds from plant sources.

\section{Materials and Methods}

\subsection{Materials}

Experimental research has focused on 5 types of hydroalcoholic extracts from Aronia, rosehip, rowan, hawthorn and grape marc, a maximum of 8 measured parameters, the number of which varies for different types of extracts. In this case study, the influencing factor was the concentration of ethyl alcohol $\left(C_{a}\right)$. For fruits of Aronia, rosehip, rowan and hawthorn were applied 5 concentrations of ethyl alcohol $(20 \%, 40 \%, 50 \%, 60 \%, 80 \%(\mathrm{v} / \mathrm{v}))$, and for grape marc-6 concentrations $(20 \%, 40 \%, 50 \%, 60 \%, 80 \%, 96 \%(\mathrm{v} / \mathrm{v}))$.

Table 1 shows the 8 measured parameters and their coding.

Table 2 shows 5 types of plant extracts and measured parameters.

\subsection{Methods}

Hermite polynomials were used. Hermite polynomials represent an important series of functions in the class of orthogonal polynomials and are solutions of Hermite's differential equation. The general term of Hermite polynomials used in probability theory is:

$$
H_{n}(x)=(-1)^{n} \mathrm{e}^{x^{2} / 2} \frac{\mathrm{d}}{\mathrm{d} x^{n}} \mathrm{e}^{-x^{2} / 2}
$$

The first 4 Hermite polynomials are: 
Table 1 . Notify the parameters and units.

\begin{tabular}{ccc}
\hline $\begin{array}{c}\text { Parameter } \\
\text { code }\end{array}$ & Parameter description & Unit \\
\hline P1 & Antioxidant activity of water-soluble substances & $\mu \mathrm{mol} \mathrm{AAE/3g}$ \\
P2 & Antioxidant activity of fat-soluble substances & $\mu \mathrm{mol} \mathrm{TE} / 3 \mathrm{~g}$ \\
P3 & Total anthocyanin content & mg ME/3g \\
P4 & Total polyphenol index & c. u. \\
P5 & Antiradical activity, DPPH, in the acidic medium & $\%$ \\
P6 & Antiradical activity, DPPH, in the basic medium & $\%$ \\
P7 & Hydrogen peroxide scavenging activity & $\%$ \\
& in the acidic medium & $\%$ \\
P8 & Hydrogen peroxide scavenging activity & in the basic medium \\
\hline
\end{tabular}

Note: AAE: ascorbic acid equivalents; TE: trolox equivalents; ME: malvidol glycoside equivalents; c.u.: conventional units; DPPH: 2,2-diphenyl-1-picrylhydrazyl.

Table 2. Types of plant extracts and codes of measured parameters.

\begin{tabular}{cc}
\hline Type of extract & Parameter code \\
\hline Aronia & P1, P2, P3, P4, P5, P6, P7, P8. \\
Rose hip & P1, P2, P4, P5, P6. \\
Hawthorn & P1, P2, P3, P4, P5, P6. \\
Rowan & P1, P2, P4, P5, P6. \\
Grape marc & P1, P2, P3, P4, P5, P6, P7, P8. \\
\hline
\end{tabular}

$$
H_{0}(x)=1 ; H_{1}(x)=x ; H_{2}(x)=x^{2}-1 ; H_{3}(x)=x^{3}-3 x
$$

where $H_{n}$ is a polynomial of degree $n$.

In order to obtain some analytical expressions, a simple nonlinear model with $y$ the resultant quantity and $x$ the factorial variable was used, the polynomial parametric model has the general form:

$$
y=\sum_{i=0}^{m} a_{m-i} x^{m-i}=a_{m} x^{m}+a_{m-1} x^{m-1}+\cdots+a_{2} x^{2}+a_{1} x+a_{0}
$$

where $m$ represents the degree of the polynomial.

Equation (3) shows the influence of the quantity $x$ on the variable $y$ and shows the interdependence between them. In this case, in Equation (3) the quantity $x$ constitutes the concentration of ethyl alcohol $\left(C_{a}\right)$, and the quantity $y$ any of the 8 measured parameters $(P 1 \ldots P 8)$.

In order to adopt the degree of the polynomial $m$ in Equation (3), 3 conditions of the mathematical model were imposed:

1) A high precision of the mathematical model, otherwise models are used on portions (as an example, between two concentrations of ethyl alcohol); 
2) The existence of experimental uncertainties requires obtaining a nominal model (with average values) and two uncertain models (upper and lower), according to the analysis of data under conditions of uncertainty [7]-[15];

3) Predictions on the values of the parameters, by deducing two predictive models, one higher and one lower [16] [17] [18] [19].

In the case of the nominal model (without uncertainties) the experimental average values at each concentration of ethyl alcohol were taken into account, and in the case of uncertain models (considering the uncertainties)-all the experimental values.

Mathematical modeling was performed in the MATLAB program (MathWorks, Inc., Natick, MA, USA).

\section{Results and Discussions}

Figure 1 and Figure 2 show the results of using the cubic Hermite polynomial to establish mathematical models for hawthorn extracts, which provide the average values of parameters $P 1, P 2, P 5$ and $P 6$ depending on the concentration of ethyl

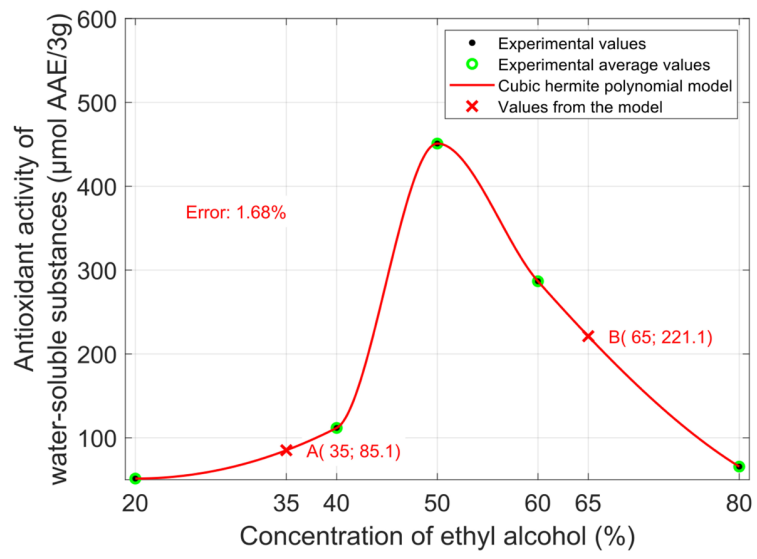

(a)

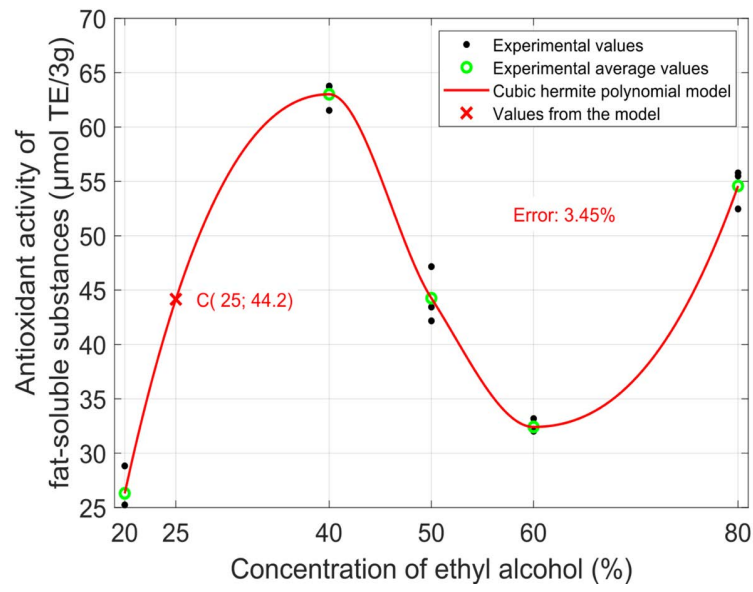

(b)

Figure 1. Polynomial mathematical models for hawthorn extracts, mean values of parameters $P 1$ (a) and $P 2$ (b) as a function of ethyl alcohol concentration. 


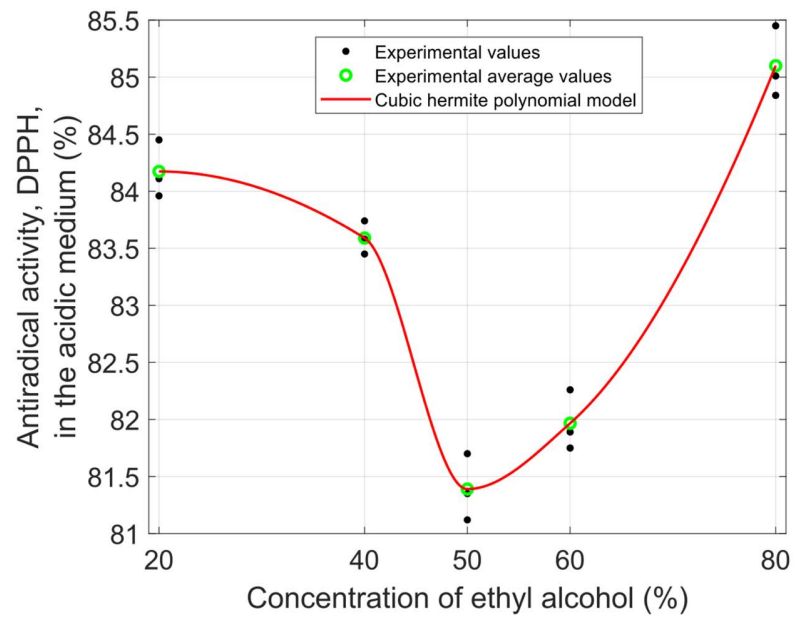

(a)

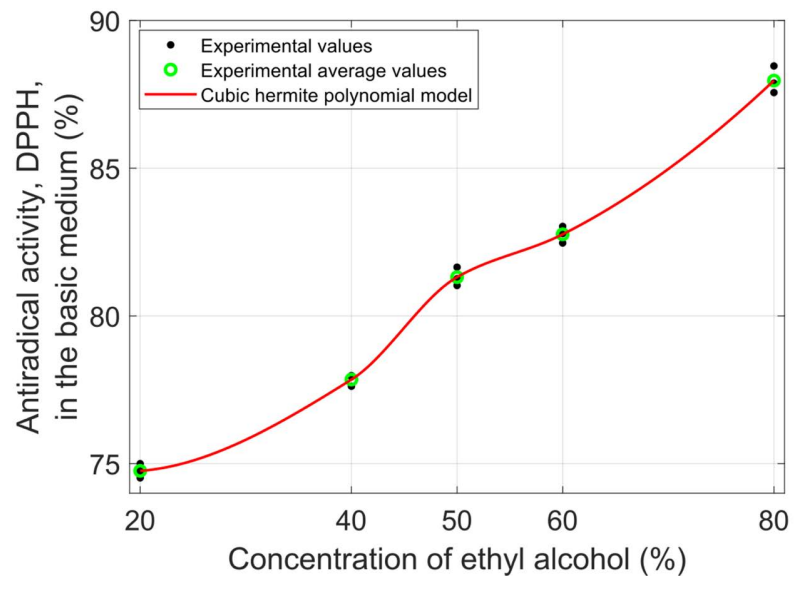

(b)

Figure 2. Polynomial mathematical models for hawthorn extracts, mean values of parameters $P 5$ (a) and $P 6$ (b) as a function of ethyl alcohol concentration.

alcohol $\left(C_{a}\right)$. In these examples as calculation data were adopted the average values of the parameters at each of the 5 concentrations of ethyl alcohol- $20 \%$, $40 \%, 50 \%, 60 \%$ and $80 \%(\mathrm{v} / \mathrm{v})$.

Figure 1(a) shows the values of parameter $P 1$ at two concentrations that are not found experimentally. It was found that at a concentration of $35 \%(\mathrm{v} / \mathrm{v})$ it is estimated that the average value of parameter $P 1$ is $85.1 \mu \mathrm{mol} \mathrm{AAE} / 3 \mathrm{~g}$ (point A); similarly, at $65 \%$ ethyl alcohol it is estimated that the mean value of parameter $P 1$ is $221.1 \mu \mathrm{mol} \mathrm{AAE} / 3 \mathrm{~g}$ (point B). Figure 1 (b) shows the average value of the parameter $P 2$ at the concentration of ethyl alcohol of $25 \%(\mathrm{v} / \mathrm{v})$, which is not found experimentally-44.2 $\mu \mathrm{mol} \mathrm{TE} / 3 \mathrm{~g}$ (point C).

Although it operates with average values, in Figure 1 and Figure 2 are presented all the experimental values of the four parameters. Figure 1 also shows the related modeling errors.

The graphs in Figure 1 and Figure 2 showed that mathematical models based on Hermite polynomials do not provide explicit analytical expressions. Instead, 
these models allow the establishment, even in the calculation algorithm, of the parameter values at any concentration of ethyl alcohol, obviously in tabular form (in addition to the corresponding graph). An example in this respect is presented in Table 3 for the average values of the 6 parameters from hawthorn extract and for ethyl alcohol concentrations with a calculation step of $5 \%$.

Figure 3 and Figure 4 show the polynomial mathematical models of the dependence between the parameter $P 1$ and the concentration of ethyl alcohol $C_{a}$ in the case of grape marc extracts.

Figure 3 and Figure 4 show that only in the case of $20^{\text {th }}$ degree polynomial (Figure 4(b)) the modeling error for the nominal model is below 2\% (value imposed for reasons of precision of the mathematical model). Moreover, only in this case the uncertain models (upper and lower 95\%) frame all the experimental data. Thus, in order to satisfy the first two conditions mentioned above (out of the 3 stated), in some cases polynomial models of a high degree can be obtained, sometimes difficult to use in practice. This aspect is obviously caused by the accentuated nonlinear character of the dependencies between the sizes.

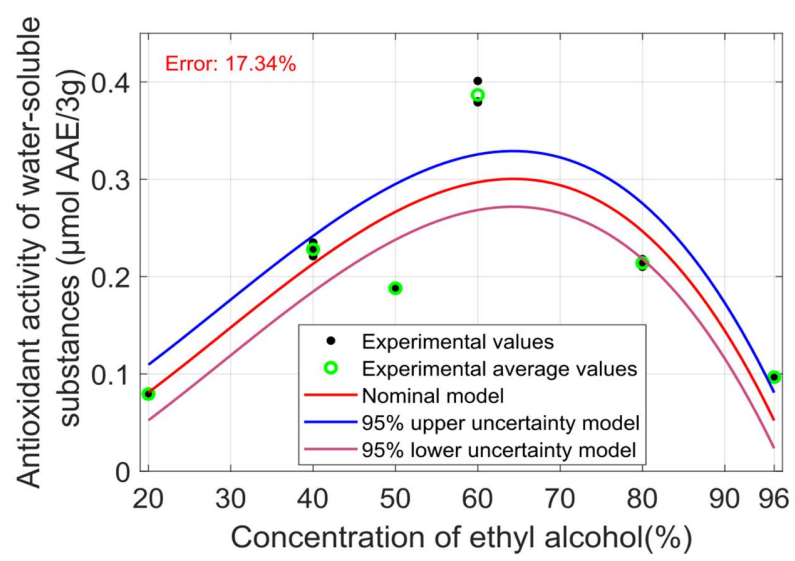

(a)

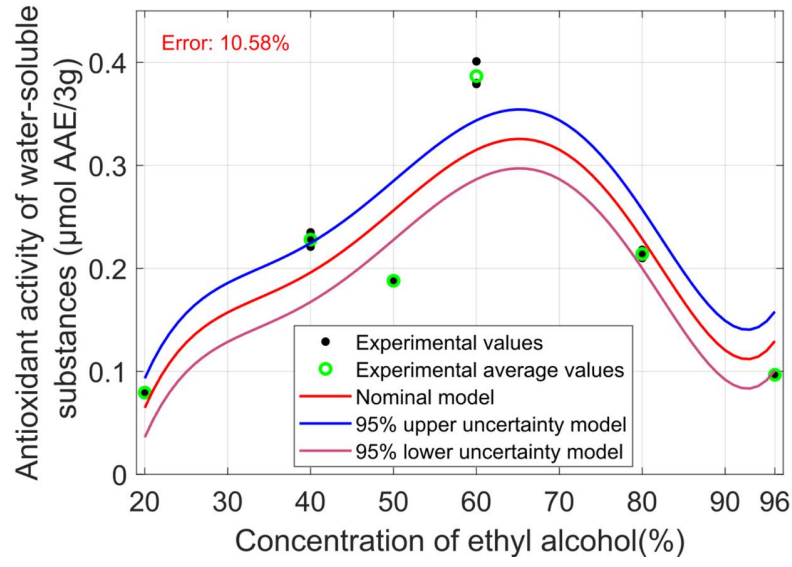

(b)

Figure 3. Polynomial mathematical models for grape marc extracts, values of parameter $P 1$ depending on the concentration of ethyl alcohol: (a) polynomial of degree 3; (b) polynomial of degree 5 . 


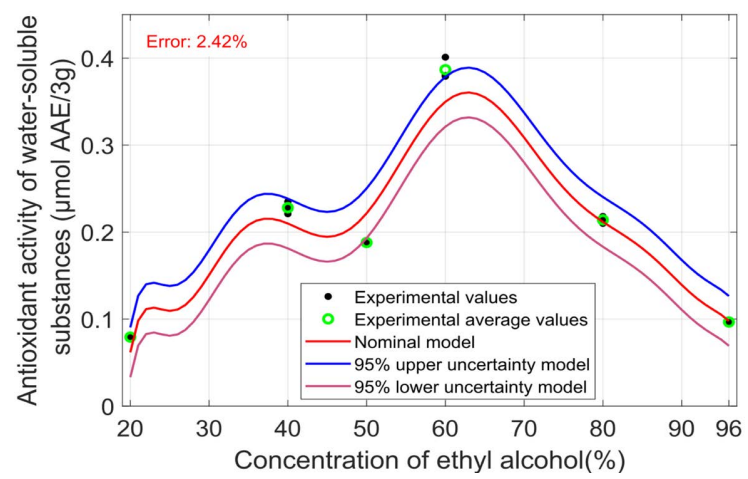

(a)

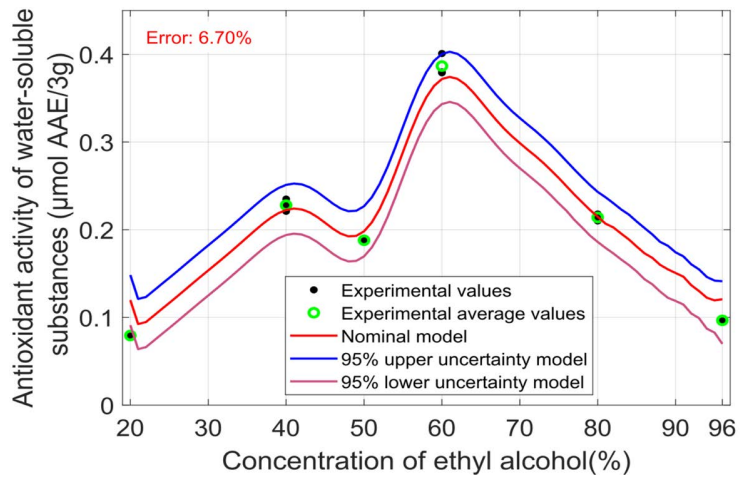

(b)

Figure 4. Polynomial mathematical models for grape marc extracts, values of parameter $P 1$ depending on the ethyl alcohol concentration: (a) polynomial of degree 10; (b) polynomial of degree 20 .

Table 3. The average values of 6 parameters from hawthorn extract for ethyl alcohol concentrations with a calculation step of $5 \%$.

\begin{tabular}{ccccccc}
\hline \multirow{2}{*}{$\begin{array}{c}\text { Ethyl alcohol } \\
\text { concentration, \% (v/v) }\end{array}$} & $P 1$ & $P 2$ & $P 3$ & $P 4$ & $P 5$ & $P 6$ \\
\cline { 2 - 7 } 20 & 51.32 & 26.30 & 0.00 & 8.27 & 84.17 & 74.76 \\
25 & 55.03 & 44.16 & 0.39 & 7.21 & 84.13 & 75.10 \\
30 & 66.24 & 55.43 & 0.60 & 6.66 & 84.02 & 75.81 \\
35 & 85.10 & 61.32 & 0.68 & 6.46 & 83.84 & 76.76 \\
40 & 111.74 & 63.01 & 0.69 & 6.43 & 83.59 & 77.85 \\
45 & 288.97 & 55.45 & 0.38 & 7.51 & 82.42 & 79.60 \\
50 & 450.91 & 44.26 & 0.07 & 9.20 & 81.39 & 81.31 \\
55 & 385.66 & 36.52 & 0.09 & 10.59 & 81.58 & 82.07 \\
60 & 286.65 & 32.41 & 0.13 & 11.37 & 81.97 & 82.76 \\
65 & 221.12 & 33.41 & 0.18 & 11.07 & 82.47 & 83.77 \\
70 & 161.03 & 36.91 & 0.26 & 10.12 & 83.18 & 84.97 \\
75 & 108.48 & 43.70 & 0.35 & 8.42 & 84.06 & 86.38 \\
80 & 65.59 & 54.58 & 0.46 & 5.87 & 85.10 & 87.97 \\
\hline
\end{tabular}


For parameters $P 1$ and $P 2$ from aronia extracts, the $5^{\text {th }}$ degree polynomial is sufficient, as shown in Figure 5.

Figure 5(b) shows the values of the parameter $P 2$ at the ethyl alcohol concentration of $35 \%(\mathrm{v} / \mathrm{v})$, non-existent experimentally. It was found that the average (nominal) value is $30.1 \mu \mathrm{mol} \mathrm{TE} / 3 \mathrm{~g}$, and the values in conditions of uncertainty are $24.9 \mu \mathrm{mol} \mathrm{TE} / 3 \mathrm{~g}$ and respectively $35.2 \mu \mathrm{mol} \mathrm{TE} / 3 \mathrm{~g}$. In each of the two graphs are presented the analytical expressions of the nominal mathematical models (with index $n$ ), as well as of the upper uncertain models (index up) and lower (index 1 ). For example, for parameter $P 1$ in Figure 5(a) the upper uncertainty model (upper curve) has the equation:

$$
P 1_{\text {up }}=-0.000014 C_{a}^{5}+0.0032 C_{a}^{4}-0.259 C_{a}^{3}+9.62 C_{a}^{2}-166.25 C_{a}+1482.58
$$

the nominal model (middle curve):

$$
P 1_{n}=-0.000014 C_{a}^{5}+0.0032 C_{a}^{4}-0.259 C_{a}^{3}+9.62 C_{a}^{2}-166.25 C_{a}+1439.12
$$

and the lower uncertainty model (lower curve):

$$
P 1_{l}=-0.000014 C_{a}^{5}+0.0032 C_{a}^{4}-0.259 C_{a}^{3}+9.62 C_{a}^{2}-166.25 C_{a}+1395.66
$$

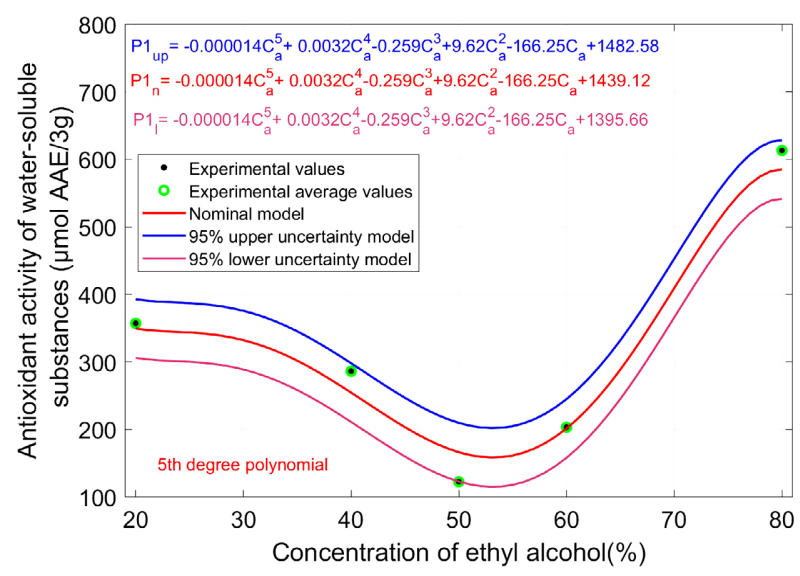

(a)

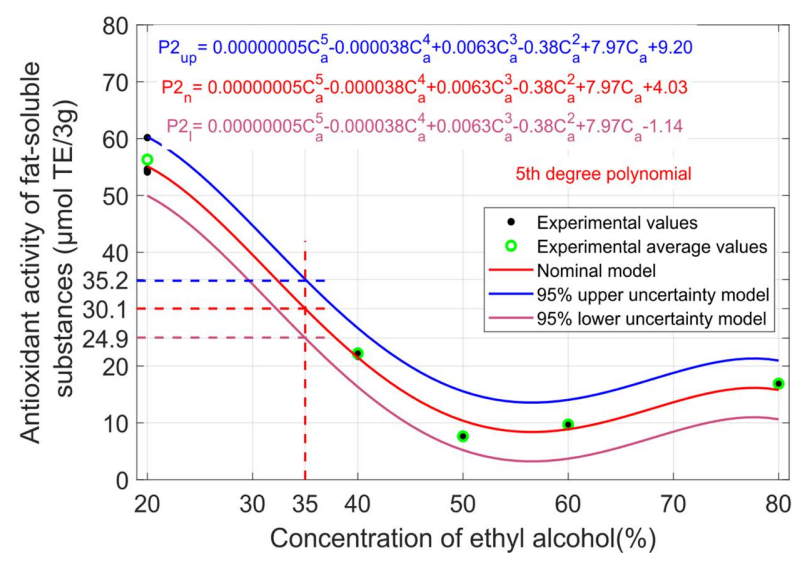

(b)

Figure 5. Polynomial mathematical models for grape marc extracts, mean values of parameters $P 1$ (a) and $P 2$ (b) as a function of ethyl alcohol concentration. 
As it can be seen, the difference between the models consists in the existence of other values of the free term, explicable by the symmetry of the confidence intervals.

The graphs in Figure 6 and Figure 7 show that for the parameter $P 3$ from the Aronia extracts a model of $5^{\text {th }}$ degree polynomial is sufficient (Figure 6(a), where the expressions of the mathematical models are also shown).

This is not the case of the parameters P4 (Figure 6(b)), P5 (Figure 7(a)) and $P 6$ (Figure $7(\mathrm{~b})$ ), which require the $20^{\text {th }}$ degree polynomial (models that are difficult to use in practice).

Figure 8 shows that for the parameter $P 7$ from the Aronia extracts a quadratic polynomial is sufficient (with the mathematical expressions in the graph), but for the parameter $P 8$ a $20^{\text {th }}$ degree polynomial is required.

Figure 8 (a) shows the expression of the nominal mathematical model:

$$
P 7_{n}=-0.00783 C_{a}^{2}+0.434 C_{a}+68.51,
$$

as well as those of uncertain models, the difference being also in the free term.

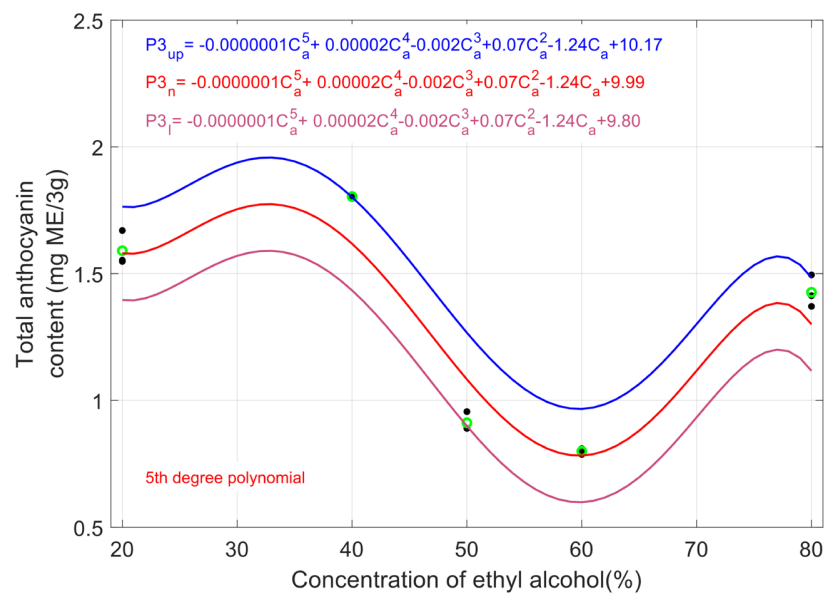

(a)

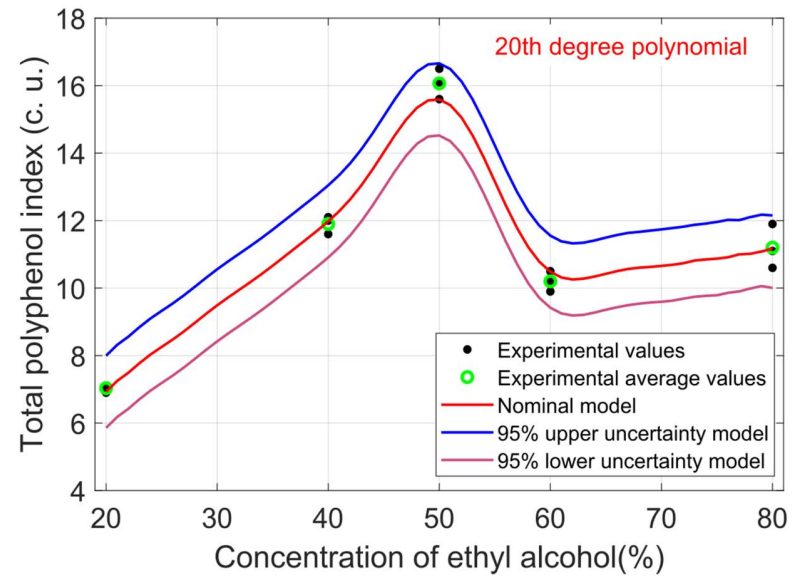

(b)

Figure 6. Polynomial mathematical models for Aronia extracts, mean values of parameters $P 3$ (a) and $P 4$ (b) as a function of ethyl alcohol concentration. 


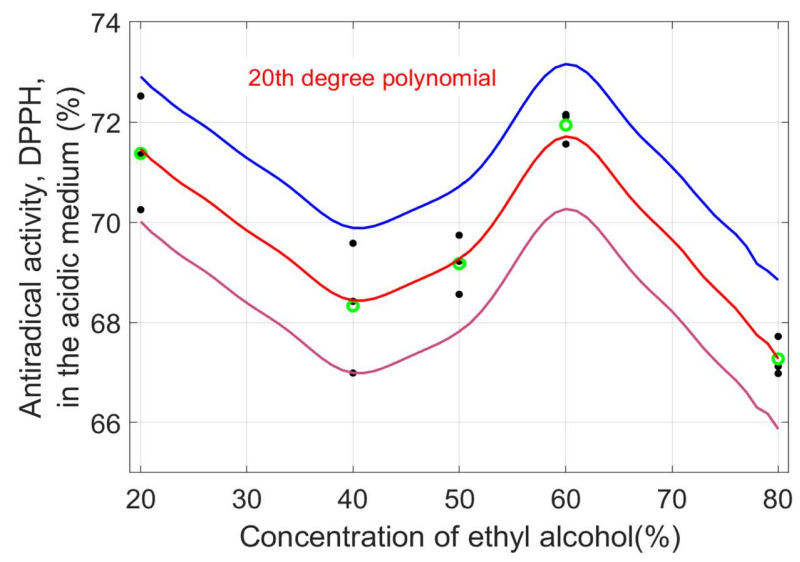

(a)

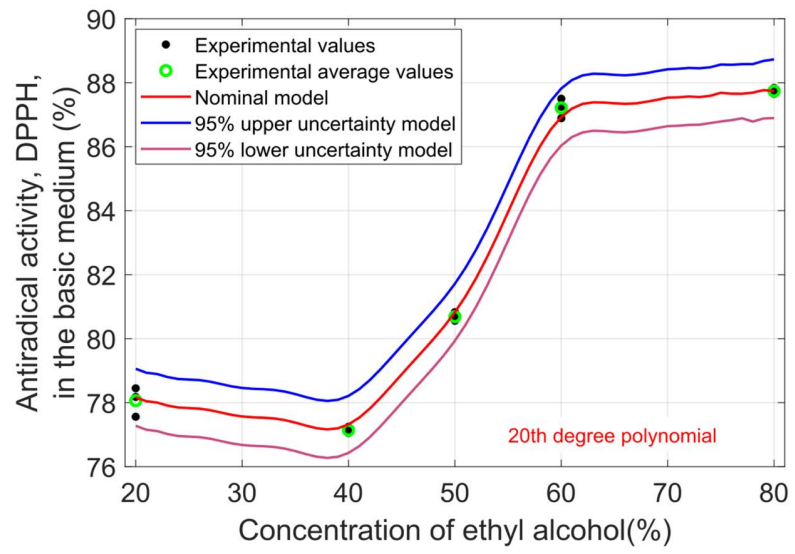

(b)

Figure 7. Polynomial mathematical models for Aronia extracts, mean values of parameters $P 5$ (a) and $P 6$ (b) as a function of ethyl alcohol concentration.

As it was found, in addition to the nominal models (of the experimental average values) were also established the uncertain models (of all the experimental data, which are located in the tires of the curves external to the nominal one). These last models were based on those previously presented in the data analysis under conditions of uncertainty.

If a high prediction is desired (the third condition of the targeted ones), then predictive mathematical models (upper and lower) must be established. An example of this is shown in Figure 9, for the case of parameter $P 2$ from rosehip extracts. Similarly, Figure 10 shows the mathematical models for parameter P5 from rowan extracts. In both of these examples $5^{\text {th }}$ degree polynomials were used.

As can be seen from the last two graphs, they contain the 5 mathematical models, compared to the previous examples being in addition 2 predictive models (index $p$ ), upper (index up) and lower (index $I$ ). As it is observed, the analytical expressions of the 5 mathematical models differ in these cases also by the value of the free term, as it was obtained in the situations only of the nominal model and of the uncertain ones. 


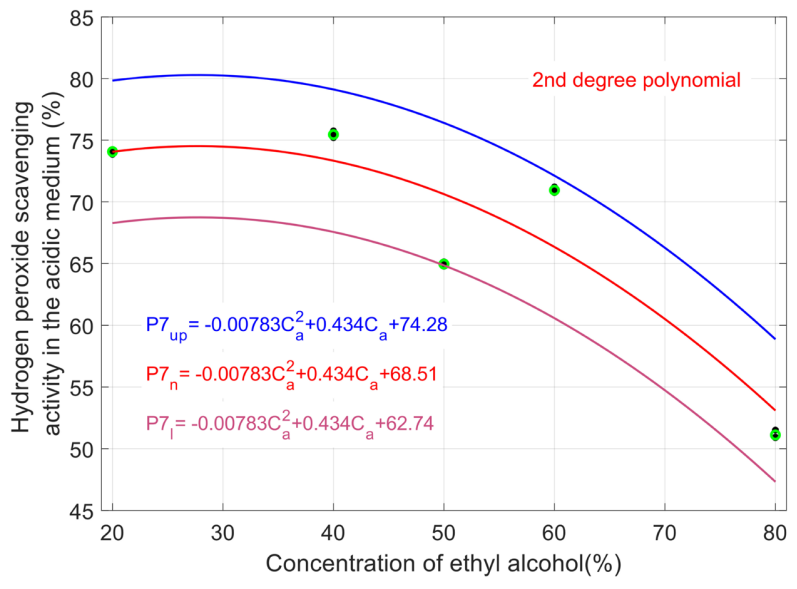

(a)

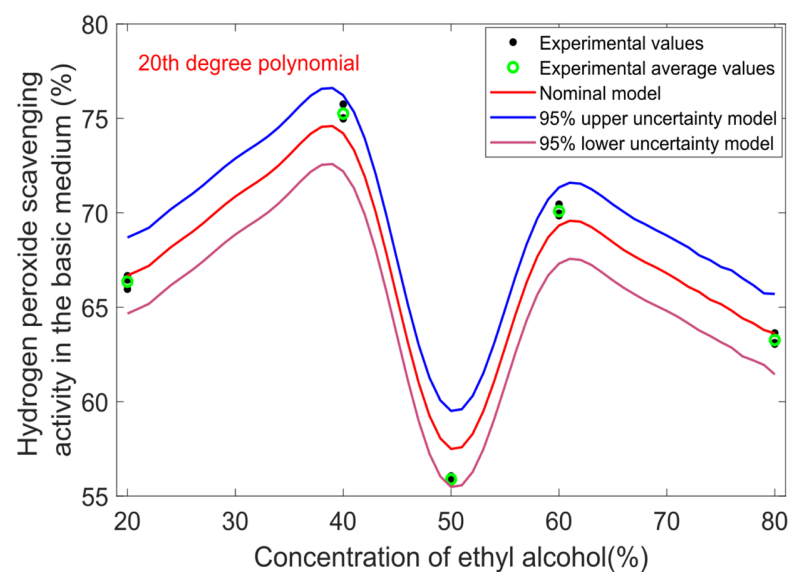

(b)

Figure 8. Polynomial mathematical models for Aronia extracts, mean values of parameters $P 7$ (a) and $P 8(\mathrm{~b})$ as a function of ethyl alcohol concentration.

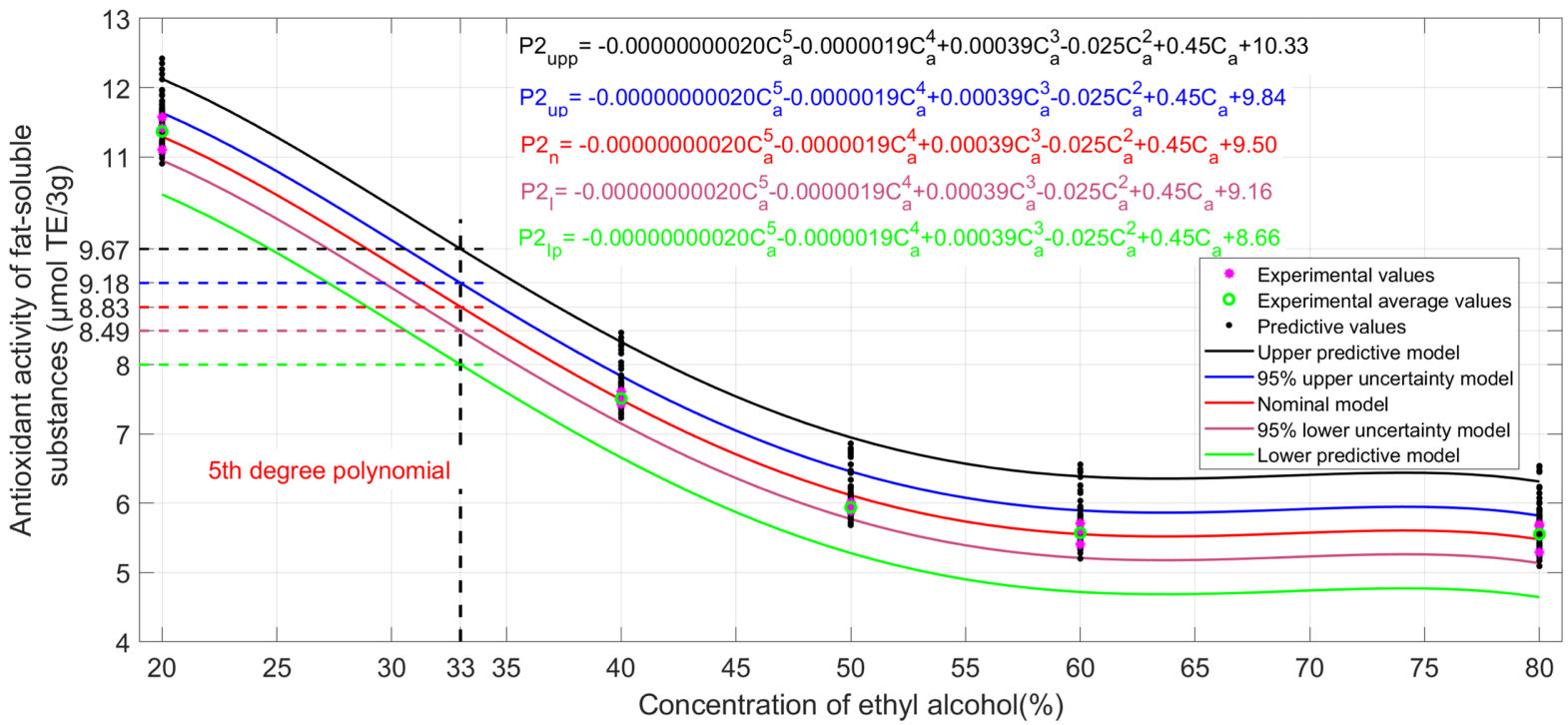

Figure 9. Polynomial mathematical models for rosehip extracts, values of parameter $P 2$ depending on the ethyl alcohol concentration. 


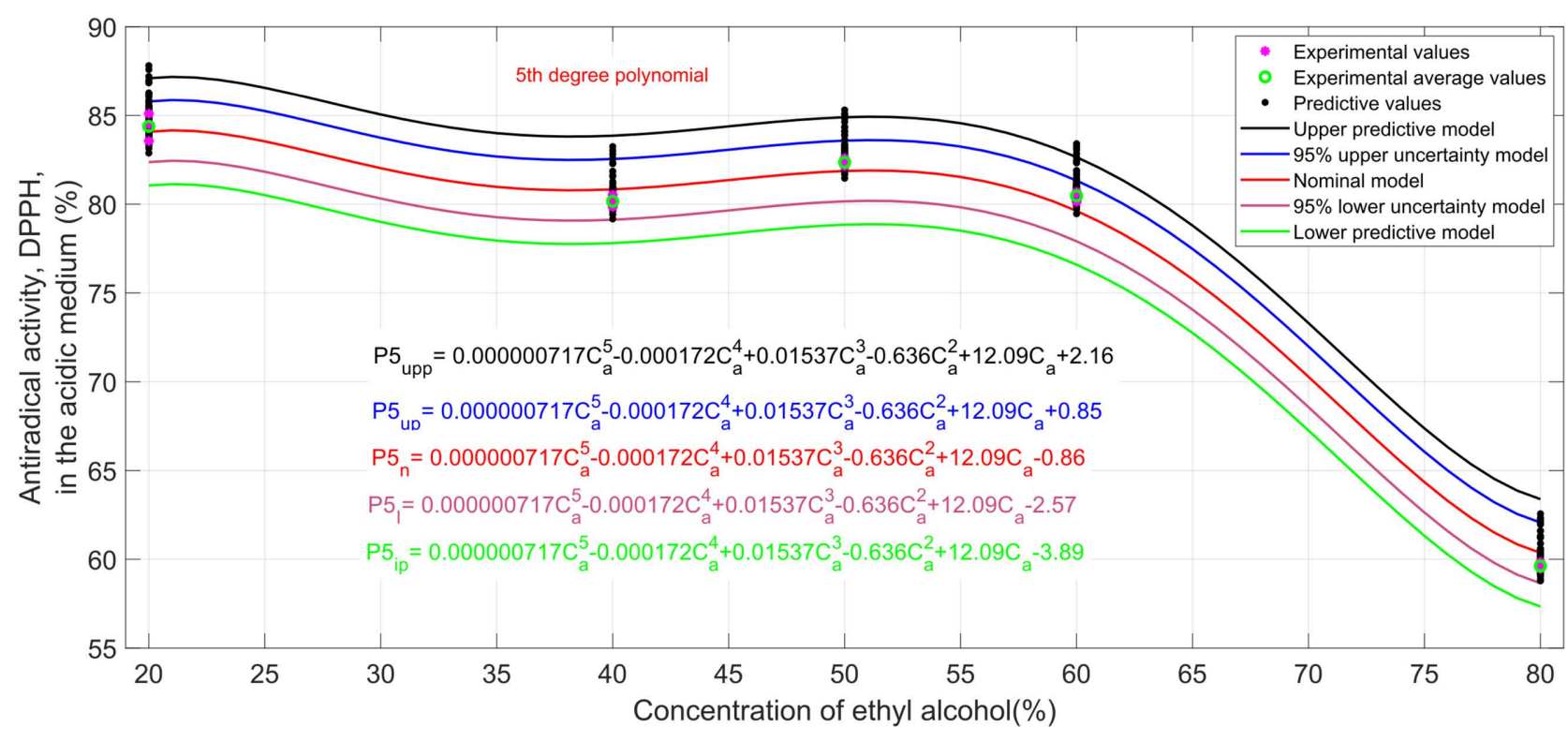

Figure 10. Polynomial mathematical models for rowan extracts, values of parameter $P 5$ depending on the ethyl alcohol concentration.

From the presented results that in some situations mathematical models are obtained on the whole sample (for all concentrations of ethyl alcohol) which require high degree polynomials, models more difficult to use in practice. One way to avoid this is to deduce polynomial mathematical models on certain intervals, i.e., between two concentrations of ethyl alcohol $C_{a}$. An example of this is shown in Figure 11 for parameters $P 1$ and $P 2$ from rosehip extracts, where cubic polynomials were used on certain intervals ( $3^{\text {rd }}$ degree), the mathematical expressions and their coefficients being shown in Figure 12.

As it can be seen from Figure 11(a), linear models cannot be used on intervals (as the line on the first interval) but only nonlinear ones, because the correlation coefficient is far from the unit value $(\rho=-0.63)$; therefore, the usage of linear models on certain intervals will provide incorrect results. Also, based on the nonlinear model, in Figure 11(a) is shown the value of the parameter $P 1=0.11$ $\mu \mathrm{mol} \mathrm{AAE} / 3 \mathrm{~g}$ at a concentration of $55 \%$ of ethyl alcohol experimentally nonexistent.

An important aspect must be emphasized here: in the analytical expressions of the mathematical models on intervals, the value of the concentration is introduced as a difference from the value of the left end of the interval, in this case 55 $-50=5$. Thus:

$$
P 1(55)=0.000122 \cdot 5^{3}-0.0018 \cdot 5^{2}+0.144=0.11 \mu \mathrm{mol} \mathrm{AAE} / 3 \mathrm{~g}
$$

From Figure 12(a), expression (1), it is found that, compared to the general relation of the mathematical model (3), this time the expression is of the form:

$$
P 1=a_{3} C_{a}^{3}+a_{2} C_{a}^{2}+a_{1} C_{a}+a_{0},
$$

for each of the 4 portions there are different values of the parameters $a_{m-i}$. For example, for the first part, with $C_{a}=20 \%-40 \%$ (Figure $12(\mathrm{a})$ ), there is a 


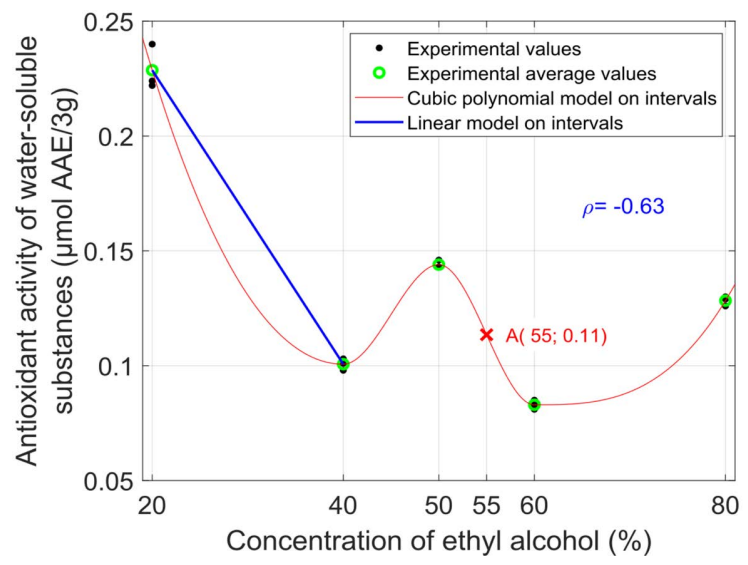

(a)

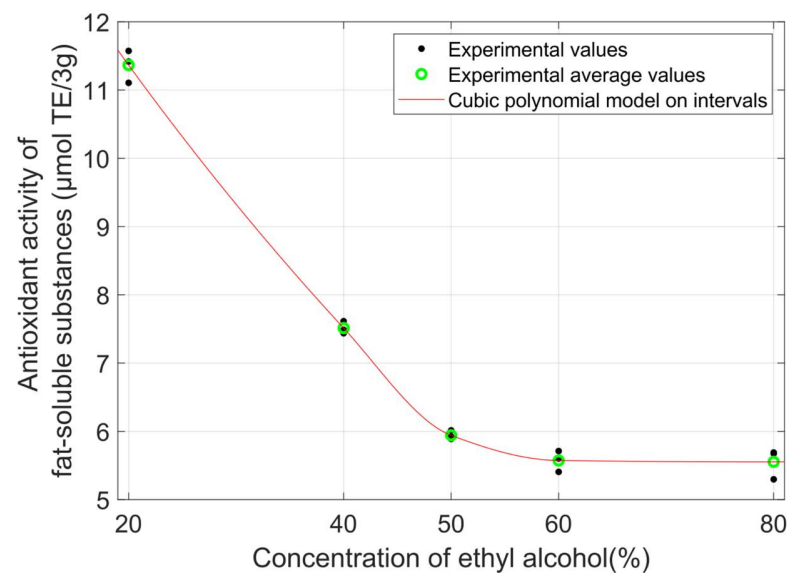

(b)

Figure 11. Polynomial mathematical models for rosehip extracts, mean values of parameters $P 1$ (a) and $P 2$ (b) as a function of ethyl alcohol concentration.

non-lacunar mathematical model (it has all the terms of the $3^{\text {rd }}$ degree polynomial):

$$
P 1_{1}=-0.0000019 C_{a}^{3}+0.000396 C_{a}^{2}-0.01356 C_{a}+0.229
$$

Instead, as can be seen from the other three graphs in Figure 12, for the last three intervals there are lacunar mathematical models, at all $a_{1}=0$, and on the last interval and $a_{2}=0$.

Similarly, Figure 13 shows the analytical expressions of the mathematical models on intervals in the case of parameter $P 2$ from rosehip extracts, which provide the average values of this parameter. As it turns out, all nominal mathematical models (with average values) are non-gaps, the coefficients having non-zero values.

Applying this algorithm, the average values of the parameters for different concentrations of ethyl alcohol are calculated with a calculation step of 5\%.

Also, in this paper, the dependencies between the measured parameters were analyzed, not only between them ( $P 1-P 8)$ and the ethyl alcohol concentration. The measured parameters are quantities that are not independent of each other. 


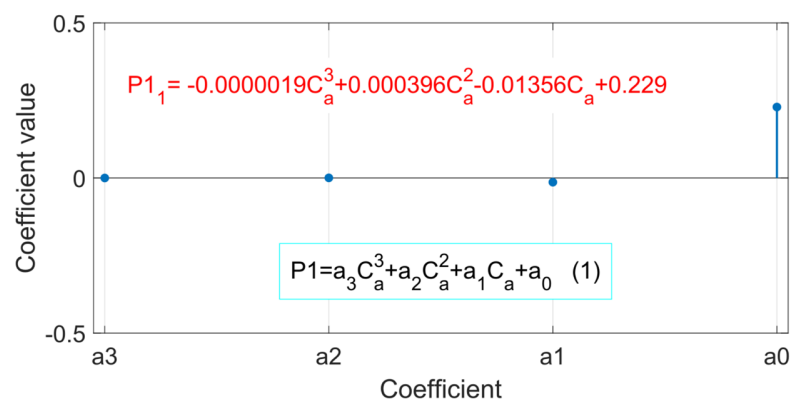

(a)

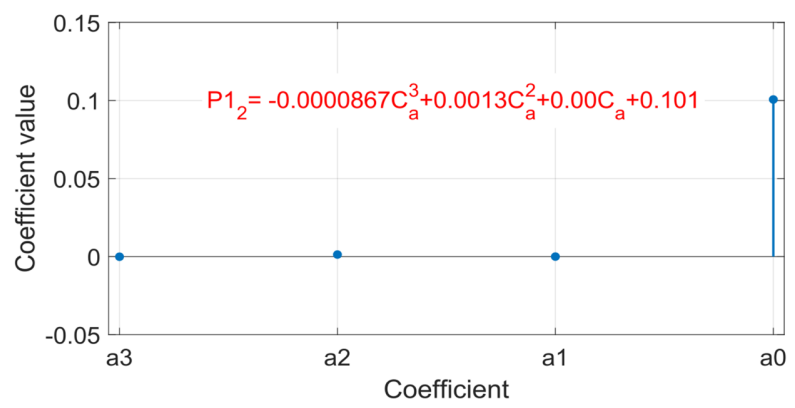

(b)

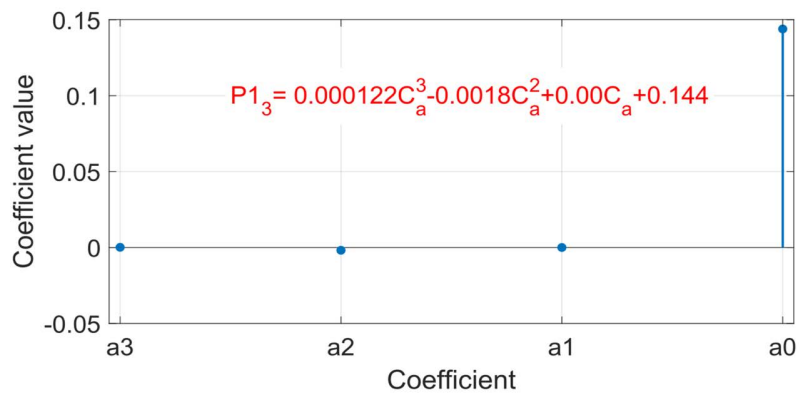

(c)

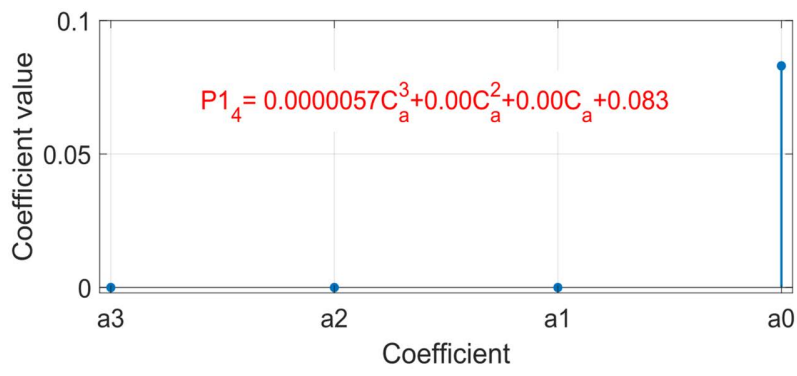

(d)

Figure 12. Coefficients of the cubic polynomial mathematical model on intervals for rosehip extracts, average values of parameter $P 1$ : (a) interval $C_{a}=20 \%-40 \%$; (b) interval $C_{a}$ $=40 \%-50 \%$; (c) interval $C_{a}=50 \%-60 \%$; (d) interval $C_{a}=60 \%-80 \%$.

An example of this is shown in Figure 14, which shows the interdependence between the concentration of ethyl alcohol, parameter $P 1$ and parameter $P 3$ from Aronia extracts, so a nominal mathematical model in the form of a multiple dependence $P 3=f\left(C_{a}, P 1\right)$. 


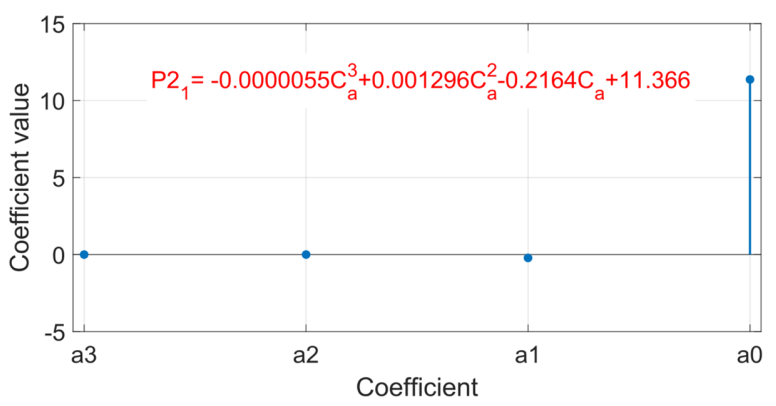

(a)

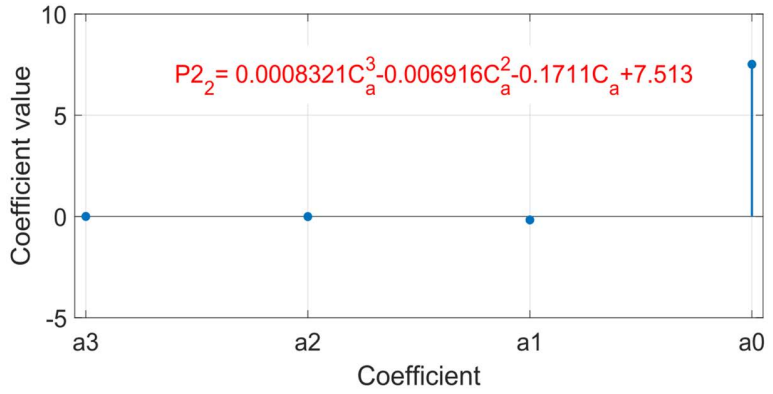

(b)

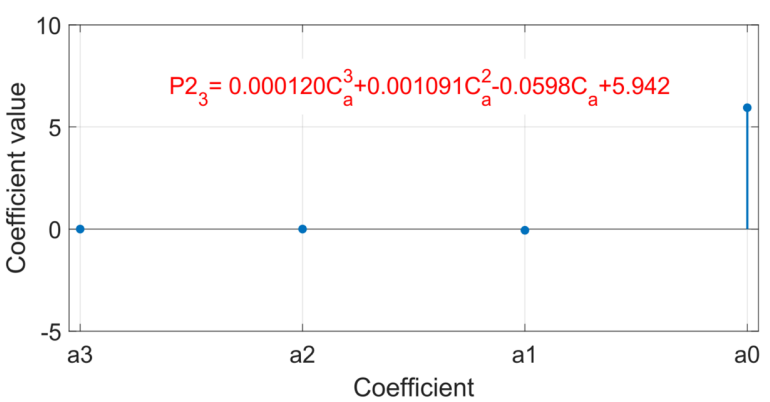

(c)

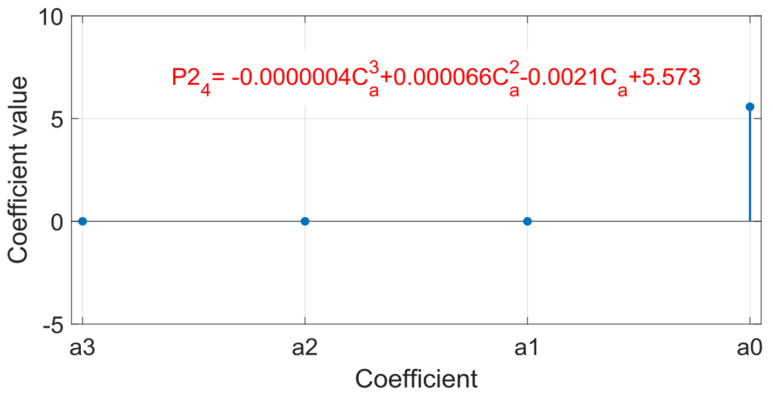

(d)

Figure 13. Coefficients of the cubic polynomial mathematical model on intervals for rosehip extracts, average values of parameter $P 2$ : (a) interval $C_{a}=20 \%-40 \%$; (b) interval $C_{a}$ $=40 \%-50 \%$; (c) interval $C_{a}=50 \%-60 \%$; (d) interval $C_{a}=60 \%-80 \%$.

The upper graphs in Figure 14 show simple dependencies of the type approached above, i.e., $P 1=f\left(C_{a}\right)$ and $P 3=f\left(C_{a}\right)$. In Figure 14(c) there is also a simple dependence, but between the two parameters, i.e., the model $P 3=f(P 1)$, whose analytical expression is plotted as a polynomial of degree 5. Finally, in 


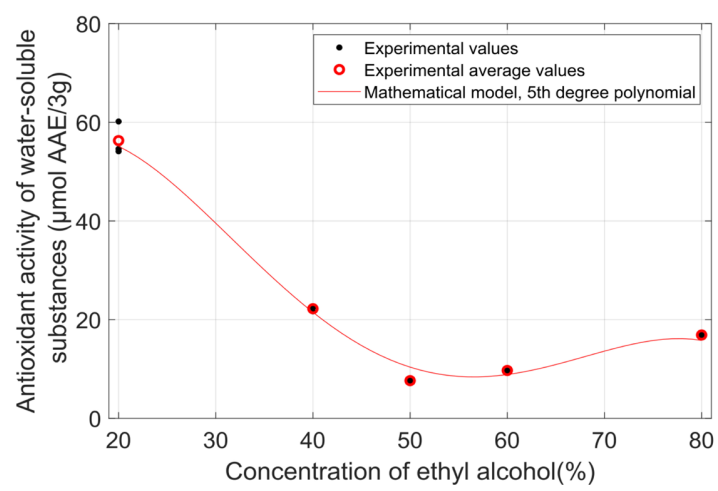

(a)

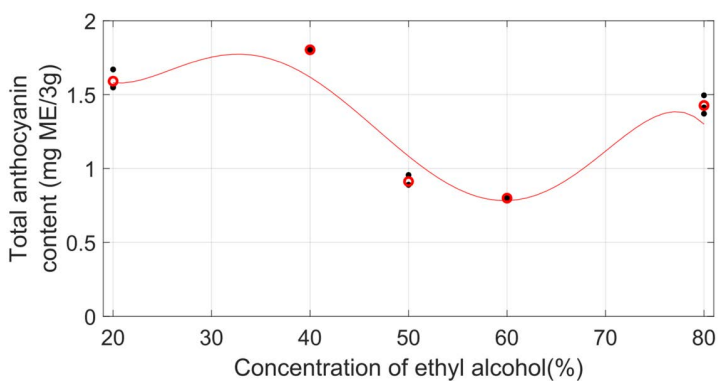

(b)

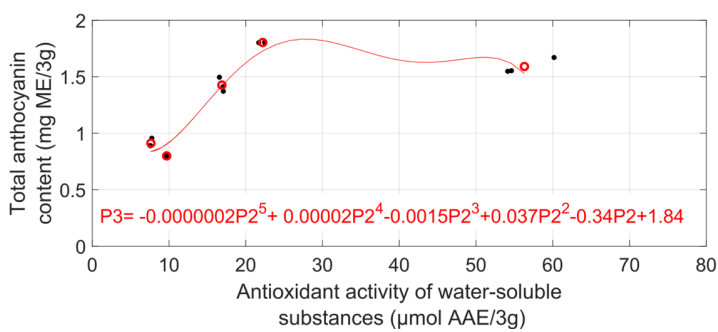

(c)

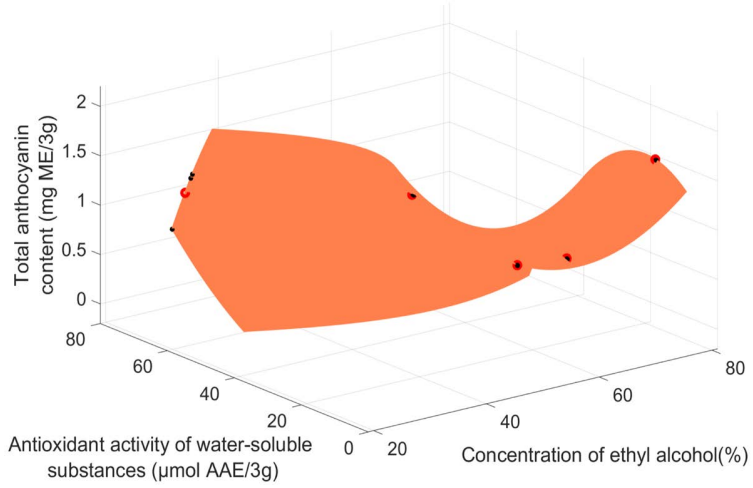

(d)

Figure 14. Mathematical model for Aronia extracts, interdependence between parameter $P 3$, ethyl alcohol concentration $\mathrm{C}_{\mathrm{a}}$ and parameter $P 1$.

Figure 14(d) it is presented only under graphic form the mathematical model sought by the form $P 3=f\left(C_{a}, P 1\right)$, obviously a surface and resumed with details in Figure 15. 


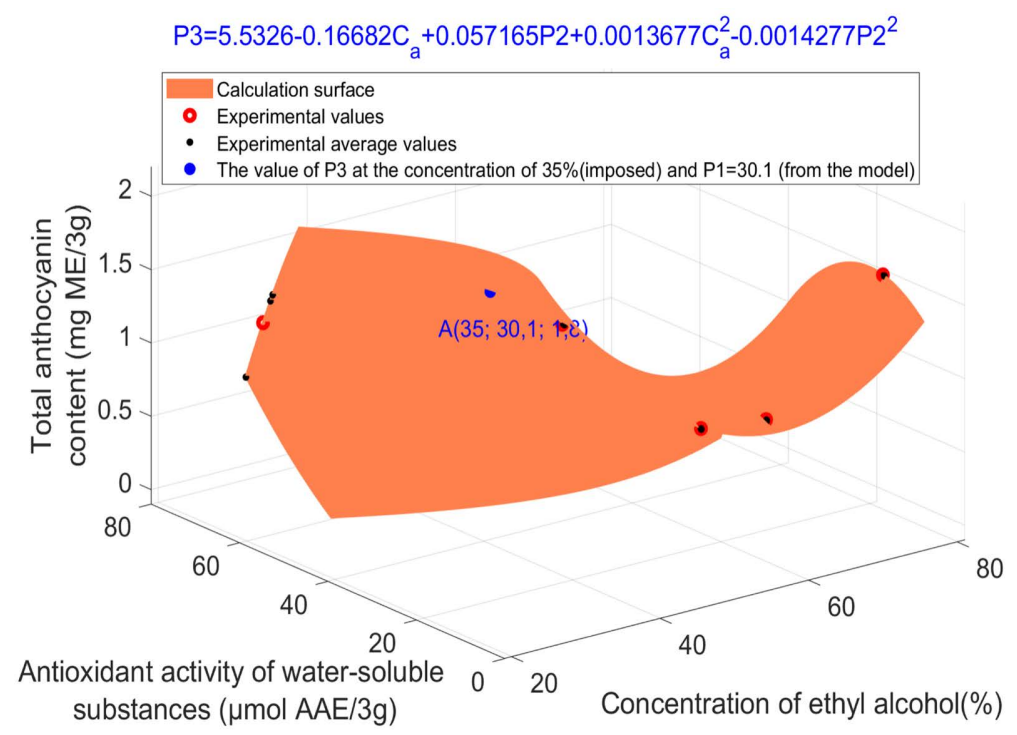

Figure 15. Mathematical model for Aronia extracts, interdependence between parameter $P 3$, ethyl alcohol concentration and parameter $P 1$.

The graph in Figure 15, which also contains the expression of the mathematical model sought, shows the calculation area, all experimental values and their averages at different concentrations of ethyl alcohol.

Also, point $\mathrm{A}$ is marked with the three coordinates obtained from the expression of the mathematical model: at the ethyl alcohol concentration of 35\%, the parameter P1 has the value $30.1 \mu \mathrm{mol} \mathrm{AAE} / 3 \mathrm{~g}$ and the parameter P3 value $1.8 \mathrm{mg} \mathrm{ME} / 3 \mathrm{~g}$.

\section{Conclusion}

The existence of few experimental data with non-zero dispersions leads to the need to establish both uncertain models, which take the experimental uncertainties into account, and predictive models, which ensure the greater credibility of the results and conclusions, are drawn. Due to the existence of nonlinear dependencies between sizes, in some situations, the classical polynomial mathematical models deduced for all concentrations of ethyl alcohol can be complicated, which are so difficult to use in practice. In the case of using polynomial models on portions (between two ethyl alcohol concentrations), analytical expressions can be obtained that are not complicated, which are so easy to use in practice. For different types of plant extracts and various measured parameters, different mathematical models are obtained, which indicates the existence of diversified phenomena and the impossibility of establishing a single model for a certain type of extract. The existence of nonlinear dependencies both between the influencing factors and the measured parameters, and between the latter, leads to the need to establish nonlinear mathematical models. In future research, polynomial mathematical models can be applied to describe the processes of extracting fat-soluble components (such as carotenoids) from plant sources. 


\section{Acknowledgements}

This work was funded through AUF-MECC Project "Intelligent Models to Improve the Training Process" running at the Technical University of Moldova.

\section{Conflicts of Interest}

The authors declare no conflicts of interest regarding the publication of this paper.

\section{References}

[1] Ghendov-Mosanu, A. (2018) Biologically Active Compounds of Horticultural Origin for Functional Foods. Tehnica-UTM, Chisinau, 236. (In Romanian)

[2] Cristea, E., Sturza, R., Jauragi, P., Niculaua, M., Ghendov-Mosanu, A. and Patras, A. (2019) Influence of $\mathrm{pH}$ and Ionic Strength on the Color Parameters and Antioxidant Properties of an Ethanolic Red Grape Marc Extract. Journal of Food Biochemistry, 43, e12788. https://doi.org/10.1111/jfbc. 12788

[3] Ghendov-Mosanu, A., Cristea, E., Patras, A., Sturza, R., Padureanu, S., Deseatnicova, O., Turculet, N., Boestean, O. and Niculaua, M. (2020) Potential Application of Hippophae rhamnoides in Wheat Bread Production. Molecules, 25, 1272. https://doi.org/10.3390/molecules25061272

[4] Ghendov-Mosanu, A., Cristea, E., Sturza, R., Niculaua, M. and Patras, A. (2020) Synthetic Dye's Substitution with Chokeberry Extract in Jelly Candies. Journal of Food Science and Technology, 57, 4383-4394. https://doi.org/10.1007/s13197-020-04475-6

[5] Ghendov-Mosanu, A., Cristea, E., Patras, A., Sturza, R. and Niculaua, M. (2020) Rose Hips, a Valuable Source of Antioxidants to Improve Gingerbread Characteristics. Molecules, 25, 5659. https://doi.org/10.3390/molecules25235659

[6] Cristea, E., Ghendov-Mosanu, A., Patras, A., Socaciu, C., Pintea, A., Tudor, C. and Sturza, R. (2021) The Influence of Temperature, Storage Conditions, pH, and Ionic Strength on the Antioxidant Activity and Color Parameters of Rowan Berry Extracts. Molecules, 26, 3786. https://doi.org/10.3390/molecules26133786

[7] Bica, A.M. (2012) Fitting Data Using Optimal Hermite Type Cubic Interpolating Splines. Applied Mathematics Letters, 25, 2047-2051. https://doi.org/10.1016/j.aml.2012.04.016

[8] Ljung, L. (1999) Model Validation and Model Error Modeling. Department of Electrical Engineering, Linkoping University, Linkoping, 24.

[9] Oberguggenberger, M. (2005) The Mathematics of Uncertainty: Models, Methods and Interpretations. In: Fellin, W., Lessmann, H., Oberguggenberger, M. and Vieider, R., Eds., Analyzing Uncertainty in Engineering, Springer-Verlag, Berlin, 51-72. https://doi.org/10.1007/3-540-26847-2 4

[10] Halpern, J.Y. (2017) Reasoning about Uncertainty. 2nd Edition, MIT Press, Cambridge, 504. https://doi.org/10.7551/mitpress/10951.001.0001

[11] Liu, B. (2015) Uncertainty Theory. Springer-Verlag, Berlin, 363. https://doi.org/10.1007/978-3-662-44354-5

[12] Taylor, B.N. and Kuyatt, C.H. (1994) Guidelines for Evaluating and Expressing the Uncertainty of NIST Measurement Results. National Institute of Standards and Technology (NIST), Technical Note 1297, Washington DC, 20. https://doi.org/10.6028/NIST.TN.1297

[13] Joint Committee for Guides in Metrology 100:2008 (2008) Evaluation of Measurement Data-Guide to the Expression of Uncertainty in Measurement. BIPM, Pavillon de 
Breteuil F-92312 Sèvres Cedex France, 134.

[14] United Kingdom Accreditation Service (2020) The Expression of Uncertainty in Testing. London, 13.

[15] EA-4/02 M (2013) Evaluation of the Uncertainty of Measurement in Calibration. European Co-Operation for Accreditation, Geneva, 75.

[16] Cho, K., Yeo, I.-K., Johnson, R.A. and Loh, W.-Y. (2001) Prediction Interval Estimation in Transformed Linear Models. Statistics Probability Letters, 51, 345-350. https://doi.org/10.1016/S0167-7152(00)00169-3

[17] Yaffee, R.A. and McGee, M. (2000) Introduction to Time Series Analysis and Forecasting. Elsevier Science, Amsterdam.

https://www.perlego.com/book/1857358/an-introduction-to-time-series-analysis-an d-forecasting-pdf

[18] Melkonian, V. (2021) Mathematical Models for a Social Partitioning Problem. American Journal of Computational Mathematics, 11, 1-22. https://doi.org/10.4236/ajcm.2021.111001

[19] Yan, S., Moreno, V., Yuan, S. and Song, B. (2018) A Mathematical Study to Gout Symptoms. Journal of Applied Mathematics and Physics, 6, 2579-2588.

https://doi.org/10.4236/jamp.2018.612215 